\title{
Regional and remote connectivity patterns in focal extratemporal lobe epilepsy
}

\author{
Wenyu Liu ${ }^{1}$, Qiang Yue ${ }^{2}$, Qiyong Gong ${ }^{2}$, Dong Zhou ${ }^{1}$, Xintong Wu ${ }^{1}$ \\ ${ }^{1}$ Department of Neurology, West China Hospital, Sichuan University, Chengdu, China; ${ }^{2}$ Department of Radiology, Huaxi MR Research Center \\ (HMRRC), West China Hospital, Sichuan University, Chengdu, China \\ Contributions: (I) Conception and design: W Liu; (II) Administrative support: Q Gong; (III) Provision of study materials or patients: D Zhou; (IV) \\ Collection and assembly of data: X Wu; (V) Data analysis and interpretation: Q Yue; (VI) Manuscript writing: All authors; (VII) Final approval of \\ manuscript: All authors. \\ Correspondence to: Dong Zhou. Department of Neurology, West China Hospital, Sichuan University, Chengdu, China. \\ Email: zhoudong66@yahoo.de; Xintong Wu. Department of Neurology, West China Hospital, Sichuan University, Chengdu, China. \\ Email: 36853839@qq.com.
}

Background: Focal epilepsy accounts for most epilepsy cases, and frontal lobe epilepsy (FLE) accounts for the largest proportion of cases of extratemporal epilepsy syndrome. The epileptogenic zone is usually not easy to locate, contributing to a lack of imaging studies. The objective of this study was to evaluate functional connectivity patterns to explore the underlying pathological mechanisms of this disorder.

Methods: Forty-three patients with focal extratemporal epilepsy [mean age \pm standard deviation (SD): $29.51 \pm 8.04$ years, 19 males] and the same number of healthy controls (mean age \pm SD: $29.56 \pm 8.02$ years, 19 males) were recruited to undergo functional magnetic resonance imaging. Mean regional homogeneity ( $\mathrm{ReHo}$ ) was measured, and regions showing significant alterations in ReHo in patients were identified to examine functional connectivity (FC). In particular, FC within the default mode network (DMN) in patients was analyzed.

Results: Patients with extratemporal lobe epilepsy showed significantly higher ReHo in the bilateral precentral gyrus, and lower ReHo in frontal-cerebellum regions than healthy controls $[\mathrm{P}<0.05$, Gaussian random field (GRF)-corrected]. FC analysis based on regions of interest showed significantly higher connectivity in the frontoparietal-insula region and lowered FC in the frontal-cerebellum regions $(\mathrm{P}<0.05$, GRF-corrected). Altered FC within DMN was also demonstrated ( $\mathrm{P}<0.05, \mathrm{GRF}$-corrected).

Conclusions: Analyses of ReHo and FC based on regions of interest suggest epilepsy-related neural networks are located mainly in frontal regions in extratemporal lobe epilepsy. These findings reveal disruptions of interactions and connectivity of large-scale neural networks and frontotemporal-cerebellar regions, suggesting connectivity-based pathophysiology.

Keywords: Extratemporal lobe epilepsy; focal cortical dysplasia (FCD); regional homogeneity; functional connectivity; default mode network (DMN)

Submitted Mar 22, 2021. Accepted for publication May 28, 2021.

doi: 10.21037/atm-21-1374

View this article at: https://dx.doi.org/10.21037/atm-21-1374

^ ORCID: 0000-0001-7101-4125. 


\section{Introduction}

Epilepsy is a common disorder marked by seizures stemming from abnormal electrical activity due to genetic or acquired causes. Focal epilepsy is a major type of epilepsy that is more common than generalized epilepsy and may lead to drug resistant epilepsy $(1,2)$. Although focal epilepsy was originally thought to arise in local regions, the disorder has been shown to involve widespread network alterations (3). Recent studies suggest that epileptogenesis in focal epilepsy results from alteration of the functional network rather than a specific region (4). Therefore, further evaluations of intrinsic neural activity and epileptic networks are increasingly important to understand the neuropathological mechanisms underlying focal epilepsy.

The development and propagation of epileptic seizures can alter structural integrity and disturb connections between structures, resulting in large-scale network instability $(5,6)$. Functional magnetic resonance imaging (fMRI) is a non-invasive, convenient approach to assess neural activity and epileptic networks in epilepsy patients (7). Alterations of connections related to the epileptogenic zone and remote functional networks have been implicated $(8,9)$. While the association between connectivity and clinical features, including epilepsy duration and seizure frequency, suggests progressive reorganization of connectivity over time, this remains poorly explored in focal extratemporal lobe epilepsy because neuroimaging studies of focal epilepsy have focused on abnormal connectivity in temporal lobe epilepsy (TLE) (10,11).

Although epilepsy is considered a disorder of neural networks, focal epilepsy is a much more widespread brain disease. Frontal lobe epilepsy (FLE) is a representative syndrome of extratemporal lobe epilepsy (12), which usually manifests as brief seizures. It is not easy to diagnose and accurately localize the epileptic region based only on clinical symptoms and an electroencephalogram (EEG) since imaging results are often negative for these patients. An EEG-fMRI study detected activation or deactivation of multiple brain regions during heterogeneous inter-ictal epileptiform discharges in FLE (13). Focal cortical dysplasia (FCD) is a frequent hidden cause of MRI-negative epilepsy $(14,15)$, particularly among patients with extratemporal lobe epilepsy or FLE (16). Fewer imaging studies have been carried out on patients with FLE than on patients with TLE, and system-wide analyses of FLE are lacking. By characterizing the brain as a complex and interconnected system, we can holistically map the network-level dysfunction underlying extratemporal lobe epilepsy (17).

Regional homogeneity ( $\mathrm{ReHo}$ ) is a metric widely employed to assess synchronization during time series, reflecting local similarity between neuronal regions. Altered values of ReHo suggest aberrant connectivity. The intrinsic activity and altered assemblies of neuronal regions may contribute to epileptogenesis in focal epilepsy. The properties of local spontaneous activity and remote functional connectivity in extratemporal lobe epilepsy have not been adequately investigated, hindering the elucidation of hubs in epilepsy-related neural networks. This is particularly important for understanding focal epilepsy, where increasing evidence suggests aberrant connections within hubs of the default mode network (DMN). Therefore, a comprehensive characterization of the functional connectivity patterns is needed to explore the underlying pathological mechanism of this disorder. Here we employed fMRI to study the ReHo and region of interest (ROI)-based functional connectivity (FC) of epilepsy patients.

We present the following article in accordance with the MDAR reporting checklist (available at https://dx.doi. org/10.21037/atm-21-1374).

\section{Methods}

\section{Subjects and clinical assessment}

This study included 43 patients with extratemporal lobe epilepsy (19 males) who attended the Comprehensive Epilepsy Center of West China Hospital, Sichuan University (Chengdu, Sichuan province) for presurgical evaluation during Jul. 2017 and Nov. 2019. In addition, 43 age- and sex-matched healthy subjects (19 males) were enrolled.

Patients with extratemporal epilepsy were diagnosed based on the 1989 International League against Epilepsy (ILAE) classification of epileptic syndromes (18). Refractory epilepsy was defined as ongoing seizures (failure of sustained seizure freedom) after using two kinds of appropriate and tolerated antiepileptic drugs, either as monotherapies or in combination (19). Frequencies of seizure attacks were classified as yearly (1-10/year), monthly (1-10/month), or daily (1-10/day). The study was conducted in accordance with the Declaration of Helsinki (as revised in 2013), and written informed consent was signed by each subject. The study protocol was approved by the Ethics Committee of West China Hospital (No. 2019: 372).

Inclusion criteria: The manifestation of MRI is FCD 
or negative; epilepsy patients; the semiology, video-EEG, and positron emission tomography (PET) suggest the extratemporal lobe as the focal origin. Exclusion criteria: Structural alteration additional to FCD including other malformations of cortical development, ganglioglioma, hippocampal sclerosis, tumor and brain injury, and pregnant women.

\section{MRI data acquisition}

Functional and structural MRI datasets were obtained at West China Hospital using a 3.0 T Siemens (Tim Trio) MRI. The resting state fMRI sensitized to alterations in BOLD signal were obtained by echo-planar imaging (EPI) sequence using the following parameters: TR 2,000 ms, echo time $30 \mathrm{~ms}$, flip angle $90^{\circ}$, slice with no slice gap and thickness of $5 \mathrm{~mm}$, the field of view $240 \times 240 \mathrm{~mm}^{2}$, voxel size $3.75 \times 3.75 \times 5 \mathrm{~mm}^{3}$, with 30 axial slices and 200 volumes. Additionally, high-resolution brain structural images were acquired using a three-dimensional T1weighted rapid gradient echo in axial orientation, and the parameters were TR $1,900 \mathrm{~ms}$, TE $2.26 \mathrm{~ms}$, FOV 256x $256 \mathrm{~mm}^{2}$, flip angle $90^{\circ}$, matrix $512 \times 512$, slice thickness $1 \mathrm{~mm}$, number of slices 176 , and acquisition time $6.4 \mathrm{~min}$. Each subject was instructed to relax their mind and body during data collection, close their eyes, and not think anything in particular. Head motion was minimized by foam padding, and scanning noise was reduced by earplugs.

\section{Data reconstruction and processing}

\section{Preprocessing}

Functional image preprocessing was carried out using SPM12 software (https://www.fil.ion.ucl.ac.uk/spm/ software/spm12) and DPRSFA V4.4 (http://rfmri.org/ DPARSF). The following steps were performed: (I) removal of the first 10 time points; (II) slice timing correction; (III) head motion correction, which saw datasets with head motion exceeding $3 \mathrm{~mm}$ in translation and $3^{\circ}$ in rotation excluded; (IV) spatial normalization. The individual T1 image was co-registered to the mean functional image, and the co-registered T1 image was segmented into gray matter (GM), white matter (WM), and cerebrospinal fluid (CSF) using a unified segmentation algorithm. The coregistered functional images were then normalized into Montreal Neurologic Institute (MNI) space and resampled to $3 \times 3 \times 3 \mathrm{~mm}^{3}$ resolution; (V) detrend; (VI) covariates regressed including the Friston-24 head motion parameters,
WM signal, and CSF signal, and adding the mean of the time series back; (VII) band-pass filter $(0.01-0.08 \mathrm{~Hz})$ was applied; (VIII) all post-processing images were smoothed with a Gaussian kernel of $4 \mathrm{~mm}$ FWHM.

\section{ReHo calculation}

ReHo was calculated for each voxel based on Kendall's coefficient of concordance (KCC) between that voxel and the 27 nearest neighbor voxels. ReHo for each voxel was normalized by dividing by the mean across all voxels in the entire brain. Band-pass filtering was not performed in preprocessing.

\section{Functional connectivity (FC) analysis}

The left medial Superior Frontal Gyrus (mSFG) was defined in the Montreal Neurological Institute (MNI) space and used as a seed in the FC analysis. We computed the mean time course of the seed as a reference to establish a correlation with the time courses of all voxels of the brain. A Fisher z-transformation was applied to all correlation maps to improve normality.

\section{Functional connectivity (FC) within DMN calculation}

To evaluate functional connectivity within DMN, 11 DMN nodes were defined as follows: a1 $(-6,52,-2)$, a2 $(-8,-56$, 26), a3 $(0,52,26)$, a4 $(-54,-54,28)$, a5 $(-60,-24,-18)$, a6 (-50, 14, -40), a7 $(0,26,-18)$, a8 $(-44,-74,32)$, a9 $(-14,-52,8)$, a10 $(-28,-40,-12)$, and a11 $(-22,-20,-26)$. Pearson's correlation was then applied between the time series of each voxel and all voxels in whole brain, then Pearson's correlation coefficients were converted to z scores using Fisher's r-to-z transformation to obtain zFC . Finally, szFC maps were obtained by smooth ing with a Gaussian kernel of 4 mm FWHM.

\section{Statistical analysis}

Two sample $t$-tests were performed to compare the group differences between HCs and patients in szFC by using DPABI V4.0 software (http://www.rfmri.org/dpabi). In the case of ReHo, the statistical threshold of multiple comparisons was set at $\mathrm{P}<0.001$ at voxel level and $\mathrm{P}<0.05$ at the cluster level, where cluster size $>18$ voxels and $\mathrm{P}$ values were two-tailed and Gaussian random field theory (GRF)corrected. FC differences between healthy controls and patients were assessed using DPABI software and the twosample $t$-test. The significance threshold was defined as $\mathrm{P}<0.001$ at the voxel level and $\mathrm{P}<0.05$ at the cluster level, 
Table 1 Clinical features of extratemporal lobe epilepsy patients and HCs

\begin{tabular}{lccc}
\hline Clinical features & Patients $(\mathrm{n}=43)$ & HCs $(\mathrm{n}=43)$ & $\mathrm{P}$ value \\
\hline Age (years) & $29.51 \pm 8.04$ & $29.56 \pm 8.02$ & $19 / 24$ \\
Sex (male/female) & $19 / 24$ & $11.28 \pm 2.61$ & 0.9786 \\
Education years & $10.28 \pm 3.20$ & $27.95 \pm 0.78$ & - \\
MMSE scores & $27.67 \pm 0.87$ & - & 0.1211 \\
Onset age (years) & $17.21 \pm 9.19$ & - & - \\
Duration (years) & $12.29 \pm 6.53$ & - & - \\
Frequency (daily/monthly/yearly) & $3 / 27 / 13$ & - \\
Seizure type (focal-only/sGTCS) & $27 / 16$ & - \\
Medication (mono/duo/poly-therapy) & $14 / 26 / 3$ & - \\
Semiology (frontal/frontal-parietal/occipital) & $37 / 5 / 1$ & - \\
FCD lesion on MRI & 11 & - \\
FCD location (frontal/parietal) & $10 / 1$ & - \\
\hline
\end{tabular}

HCs, healthy controls; sGTCS, secondarily generalized tonic-clonic seizures; MMSE, mini-mental state examination; FCD, focal cortical dysplasia; MRI, magnetic resonance imaging.

where $\mathrm{P}$ values were GRF-corrected. The threshold of resultant $\mathrm{T}$-maps of szFC were defined as $\mathrm{P}<0.05$ at the voxel level and $\mathrm{P}<0.05$ at the cluster level (GRF-corrected).

\section{Results}

\section{Subject characteristics}

A total of 43 extratemporal epilepsy subjects were enrolled in this study (19 males, mean $\pm \mathrm{SD}, 29.51 \pm 8.04$ years), with a mean age at seizure onset of $17.21 \pm 9.19$ years. All included patients were defined as drug-resistant epilepsy based on the definition listed above. Most patients (30/43) had at least monthly seizures despite receiving two kinds of antiepileptic strategies (Table 1).

In this extratemporal epilepsy cohort, 11 patients showed specific imaging characteristics of FCD; with 10 in the frontal lobe and one in the parietal lobe. While most remaining patients were MRI-negative, we speculate they had subtle lesions because semiology, video-EEG, and PET suggested a focal origin in the frontal lobe (37 patients) or frontal-parietal lobe (6 patients).

\section{Disrupted intrinsic activity}

The patient group showed higher ReHo in the bilateral precentral gyrus and lower ReHo in frontal-cerebellum regions (Table 2 and Figure 1).

\section{ROI-based functional connectivity analysis}

None of the regions where ReHo was significantly altered correlated with clinical features such as epilepsy duration or seizure frequency. We used the left medial Superior Frontal Gyrus (mSFG) as a seed for more detailed analysis. This region was the largest to show altered $\mathrm{ReHo}$ and is a critical hub in the DMN and in extemporal lobe epilepsy. Patients showed significantly higher FC in frontoparietal-insula regions but significantly lower FC in frontal-cerebellum regions (Figure 2, Table 3).

\section{FC within DMN}

FC within the DMN differed significantly between groups of patients and healthy controls (Figure 3).

\section{Discussion}

The present study provides evidence of the presence of specific functional alterations in extratemporal epilepsy. We applied one of the most used MRI metrics, ReHo, to assess synchronicity of local intrinsic activity and detected higher ReHo in the bilateral precentral gyrus but lower ReHo in the mSFG and MFG of patients compared to 
Table 2 Regions showing abnormal ReHo in extratemporal lobe epilepsy patients compared with HCs

\begin{tabular}{|c|c|c|c|c|c|}
\hline Brain regions & \multicolumn{3}{|c|}{ Peak MNI coordinates } & Voxel size & $\mathrm{T}$ \\
\hline \multicolumn{6}{|c|}{ Regions with higher ReHo in patients relative to HCS } \\
\hline Precentral_R & 33 & -15 & 60 & 84 & 5.19 \\
\hline Precentral_L & -33 & -15 & 60 & 18 & 5.19 \\
\hline Frontal_Sup_Medial_L & -6 & 54 & 12 & 87 & -4.59 \\
\hline Cerebellum_Crus2_L & -21 & -78 & -33 & 83 & -5.01 \\
\hline Frontal_Mid_R & 45 & 48 & 18 & 23 & -4.76 \\
\hline Fusiform_L & -30 & -63 & -9 & 18 & -4.22 \\
\hline
\end{tabular}

ReHo, regional homogeneity; MNI, montreal neurological institute; HCs, healthy controls; Precentral_R, right Precental gyrus; Precentral_ L, left Precental gyrus; Frontal_Sup_Medial_L, left medial Superior frontal gyrus; Frontal_Mid_R, right Middle frontal gyrus; Fusiform_L, left Fusiform gyrus; Cerebellum_Crus2_L, left Inferior Cerebellum.

controls. When ROIs were defined based on the mSFG, we observed higher remote $\mathrm{FC}$ in the frontoparietal-insula regions but lower remote $\mathrm{FC}$ in the frontal-cerebellum region of patients. These results demonstrate that patients with extratemporal lobe epilepsy have remote functional alterations that may potentially affect the entire brain and further demonstrate that BOLD metrics may be useful for characterizing these alterations.

Epilepsy is a complicated disorder of altered neural networks. Different epilepsy syndromes are related to widespread disruption of neural networks, which has been linked to focal epileptic discharges. Further, diverse changes in neural network connectivity have also been described in response to various treatments in focal epilepsy. Studies have identified regions showing altered connectivity between patients with well-controlled epilepsy and those with treatment-resistant epilepsy $(20,21)$. In this study, all included patients were medically refractory.

Even though focal epilepsy was originally thought to originate in specific locations within one hemisphere, the disease is increasingly considered to involve widely distributed epileptic networks (22). Consistent with this concept, studies have observed complex associations among intrinsic activity, structural pathology, and network-level or whole-brain connectivity in focal epilepsy (23). For example, fMRI has revealed aberrant connections between frontal regions and wider brain zones (parietal-temporalcerebellum regions) in FLE (24), and abnormal functional connectivity in FLE appears to be widespread within the frontal regions (25).

$\mathrm{ReHo}$ has been widely recognized as a metric for measuring an intrinsic neural activity and detecting similarities in activity between different regions that may form part of an epileptogenic zone. The abnormal ReHo in the precentral gyrus is also in line with clinical hyper motor features. Our findings support the idea that seizures in FLE originate in sensorimotor regions extending across the cerebellum and frontal lobe regions. FLE patients were previously reported to exhibit impaired structural and effective connectivity involving the frontal and cerebellum regions (26). Another FLE study (27) using a novel metric of FOCA to measure the spatiotemporal consistency of adjacent local activity demonstrated higher FOCA in the left precentral gyrus yet lower FOCA in the cerebellum and precuneus. The FOCA findings are consistent with our results here to a large extent, but some differences may reflect that ReHo focuses on regional functional synchronization. At the same time, FOCA is more sensitive to local spatiotemporal consistency and the stability of activity states. Many studies have implicated altered synchronization and stability of intrinsic activity in extratemporal lobe epilepsy.

We observed significantly higher FC in the frontalinsula regions of the patient group but lowered FC in the cerebellum. Most of our patients were considered to have FLE based on evaluating their epileptic features, ictal EEG, and metabolic examinations. Previous research has linked alterations in frontoparietal-insula regions, and 

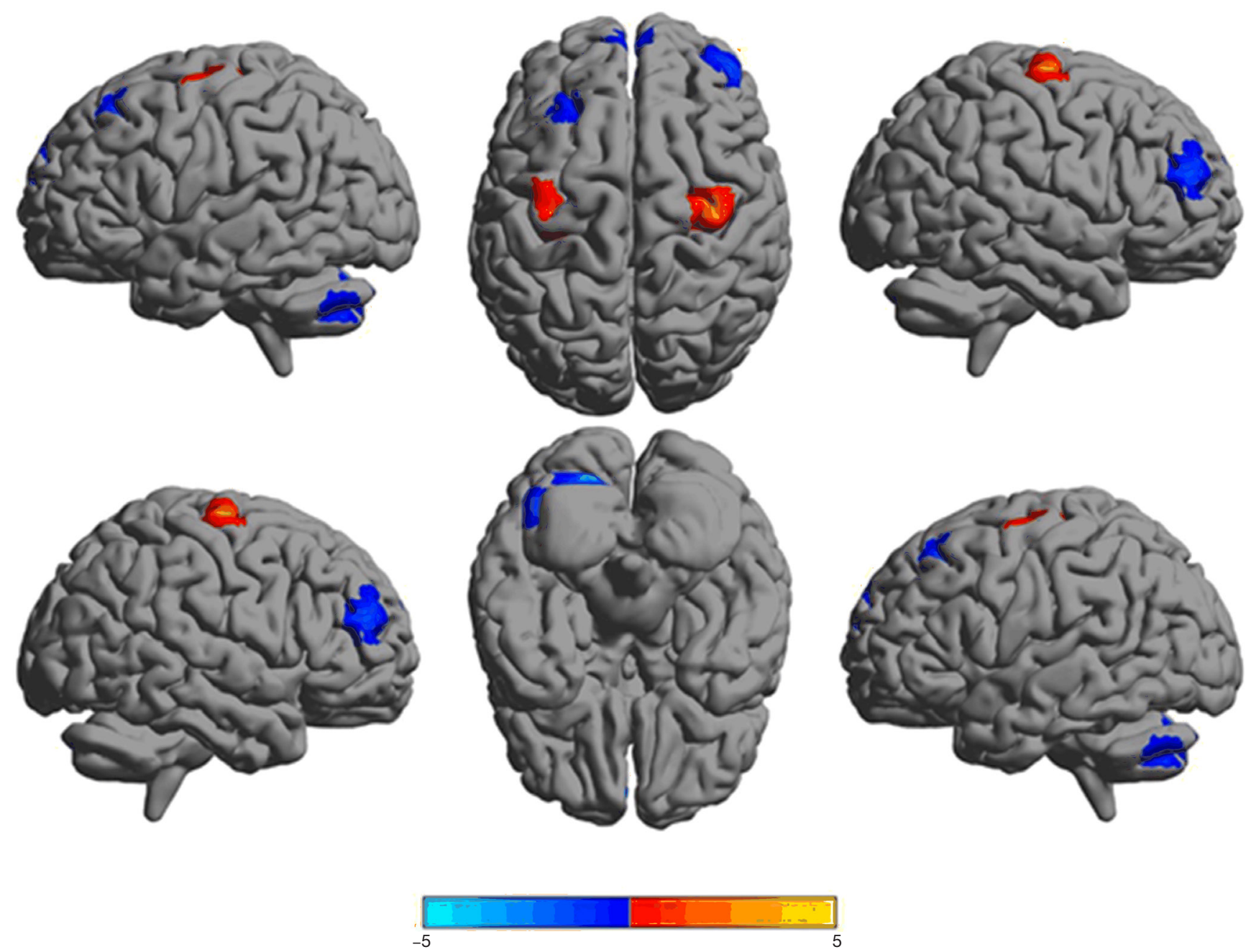

Figure 1 Brain regions showing significant differences in regional homogeneity (ReHo) between patients with extratemporal lobe epilepsy and healthy controls. The patient group showed higher ReHo in the bilateral precentral gyrus, but lower ReHo in frontal-cerebellum regions. Color bars indicate $\mathrm{T}$ values, where red indicates higher connectivity and blue indicates lower connectivity. ReHo was compared between brain regions using two-sample $t$-test, and differences associated with $\mathrm{P}<0.05$ (Gaussian random field-corrected) were considered significant.

the cerebellum to the altered FC in epilepsy, and patients with nocturnal FLE were reported to have higher uptake in frontal regions based on PET (28,29). A dynamic FC study of FLE patients revealed significantly disturbed communication of the frontoparietal system (30), and frontoparietal circuits are also involved in the cortical motor system (31). Our observation of abnormal interregional FC suggests the remote neural network is altered in extratemporal lobe epilepsy. The involvement of these brain regions is consistent with the semiology of hyper motor activity and dystonic posture during seizures, which may also be related to epileptic discharges and impaired cognition.

FLE usually manifests as a bilateral asymmetric tonic or hyper motor activity, as observed in our patients, and this may reflect impairment in both the cerebellum and frontal lobe (26). The DMN is a well-characterized epilepsyrelated network (32), and abnormal FC within the DMN is an indicator of impaired network integrity associated with treatment resistance and cognitive decline. A study of patients with idiopathic generalized epilepsy (IGE) showed reduced DMN connectivity, and drug-resistant 

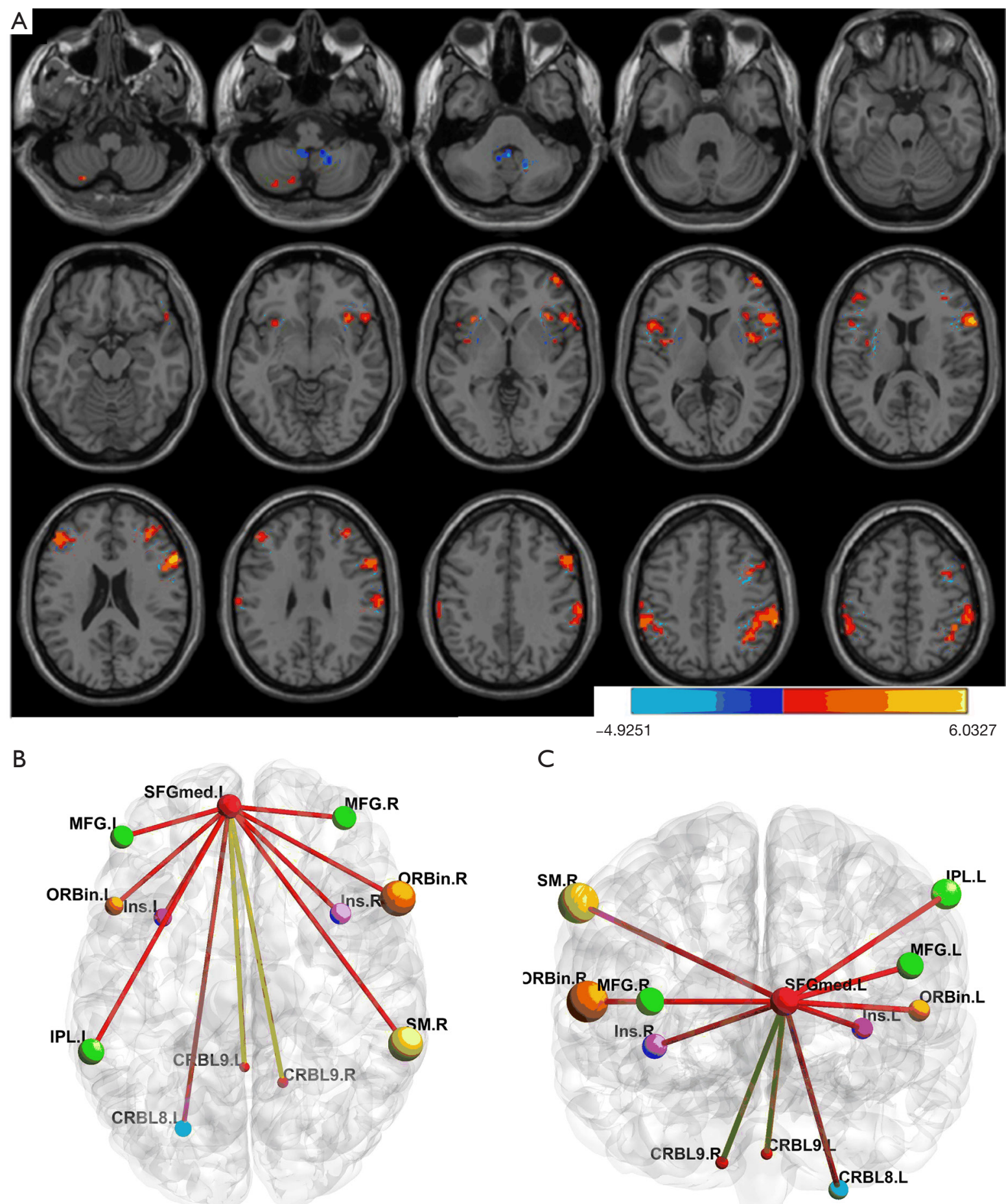

C

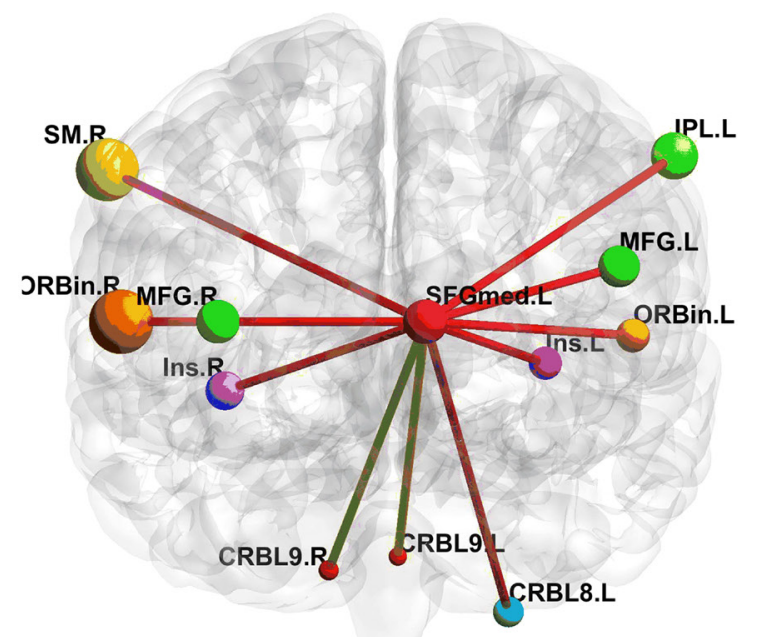

Figure 2 Altered FC involving the mSFG between patients with extratemporal lobe epilepsy and healthy controls. Patients showed significantly higher FC in frontoparietal-insula regions but significantly lower FC in the frontal-cerebellum regions. (A) Axial imaging of FC alterations. Color bars reflect T values. (B,C) Three-dimensional reconstruction of FC alterations. Red indicates higher connectivity and yellow indicates lower connectivity. Two-sample $t$-test (GRF-corrected) has been performed and $\mathrm{P}<0.05$ was considered statistically significant. mSFG, medial superior frontal gyrus; FC, functional connectivity; GRF, Gaussian random field. 
Table 3 Regions showing abnormal interregional FC in the patient group compared with HCs

\begin{tabular}{|c|c|c|c|c|c|c|}
\hline Seed & Connected area & \multicolumn{3}{|c|}{ Peak MNI coordinates } & Voxel size & $\mathrm{T}$ \\
\hline \multicolumn{7}{|c|}{ Regions with higher FC in patients relative to HCS } \\
\hline \multirow[t]{6}{*}{ Frontal_Sup_Medial_L } & Frontal_Inf_Oper_R & 60 & 18 & 12 & 288 & 6.03 \\
\hline & SupraMarginal_R & 63 & -39 & 45 & 263 & 5.11 \\
\hline & Frontal_Mid_L & -48 & 42 & 24 & 73 & 4.39 \\
\hline & Frontal_Mid_R & 36 & 42 & 21 & 48 & 4.56 \\
\hline & Frontal_Inf_Oper_L & -51 & 15 & 9 & 46 & 5.09 \\
\hline & Cerebelum_8_L & -24 & -72 & -51 & 38 & 4.59 \\
\hline \multicolumn{7}{|c|}{ Regions with lower FCS in patients relative to HCS } \\
\hline \multirow[t]{2}{*}{ Frontal_Sup_Medial_L } & Cerebelum_9_R & 15 & -54 & -42 & 20 & -4.93 \\
\hline & Cerebelum_9_L & 0 & -48 & -39 & 18 & 4.31 \\
\hline
\end{tabular}

FC, functional connectivity; HCs, healthy controls; MNI, Montreal Neurological Institute; L, left; R, right; Frontal_Sup_Medial_L, left medial Superior frontal gyrus; Frontal_Inf_Oper_R, right inferior frontal gyrus, opercular part; SupraMarginal_R, right Supramarginal gyrus; Parietal_Inf_L, left Inferior parietal gyrus; Frontal_Mid_L, left Middle frontal gyrus; Frontal_Mid_R, right Middle frontal gyrus; Frontal_Inf_ Oper_L, left Inferior frontal gyrus, opercular part; Insula_R, right Insula; Frontal_Mid_R, right Middle frontal gyrus; Cerebelum_8_L, left Inferior Cerebellum; Cerebelum_9_R, right Inferior Cerebellum; Cerebelum_9_L, left Inferior Cerebellum.

A

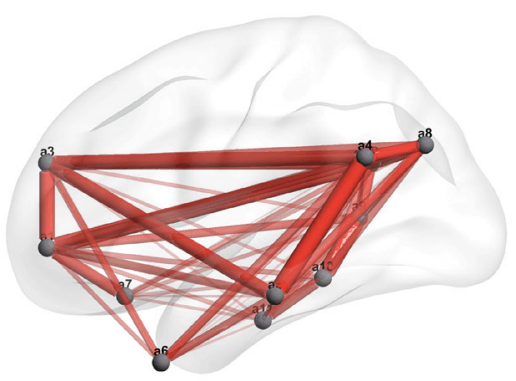

B

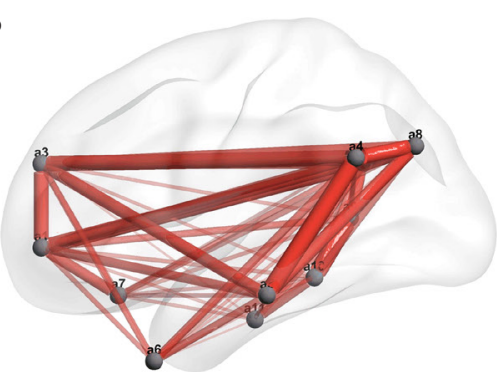

C

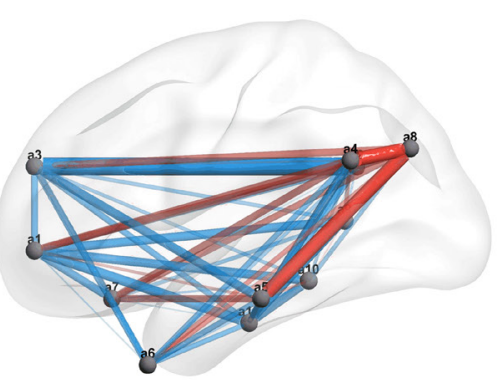

Figure 3 Alterations in functional connectivity in regions of interest (ROIs) (a1-a11) within the default mode network (DMN). (A) Connectivity within the DMN in patients. (B) Connectivity within the DMN in healthy controls. (C) Differences in connectivity between patients and healthy controls. Red indicates higher connectivity in patients and blue indicates lower connectivity. ROI descriptions: L: Left; a1. Frontal_Med_Orb_L (Superior frontal gyrus, medial orbital), a2. Precuneus_L (Precental gyrus), a3. Frontal_Sup_Medial_L (Superior frontal gyrus, medial), a4. Angular_L (Angular gyrus), a5. Temporal_Inf_L (Inferior temporal gyrus), a6. Temporal_Pole_Mid_L (Temporal pole: middle temporal gyrus), a7. Rectus_L (Gyrus rectus), a8. Occipital_Mid_L (Middle occipital gyrus), a9. Calcarine_L (Calcarine fissure and surrounding cortex), a10. Fusiform_L (Fusiform gyrus), a11. ParaHippocampal_L (Parahippocampal gyrus).

patients showed even lower DMN connectivity than drugresponsive ones (33). Lower connectivity within the DMN was also reported in TLE (34). In the absence of seizure patients, the DMN can show altered integration related to awareness and cognition, even in the absence of interictal epileptic activity or seizures (35). Another FLE study 
revealed an association between abnormality in frontalDMN zones and cognitive deficit (27). Decreased DMN connectivity is thought to reflect impaired consciousness, which is a common symptom in patients with extratemporal lobe epilepsy.

There are several limitations to this study. Firstly, the sample size was relatively small, and few patients underwent surgery. The heterogeneity among patients with extratemporal lobe epilepsy cannot be neglected, even though we included subjects that appeared to have FLE. Secondly, hidden, lesion-like, subtle FCD cannot be detected by 3-T MRI, and such FCD can influence FC and network patterns in extratemporal lobe epilepsy. This is one reason why relatively few studies have examined FC in this disorder. Thirdly, the use of antiepileptic drugs may have confounded our FC analysis. Future research should verify and extend our findings to elucidate FC patterns comprehensively in this disease.

\section{Acknowledgments}

Funding: This study was supported by the National Natural Science Foundation of China (Grant No. 81901320), China Postdoctoral Science Foundation funded project (Grant No. 2019M653425), Sichuan Science and Technology Program (Grant No. 2020YJ062), Chengdu Science and Technology Bureau Program (2019-YF09-00215-SN) and Post-doctor Research Project, West China Hospital, Sichuan University (Grant No. 2018HXBH075).

\section{Footnote}

Reporting Checklist: The authors have completed the MDAR reporting checklist. Available at https://dx.doi. org/10.21037/atm-21-1374

Data Sharing Statement: Available at https://dx.doi. org/10.21037/atm-21-1374

Conflicts of Interest: All authors have completed the ICMJE uniform disclosure form (available at https://dx.doi. org/10.21037/atm-21-1374). The authors have no conflicts of interest to declare.

Ethical Statement: The authors are accountable for all aspects of the work in ensuring that questions related to the accuracy or integrity of any part of the work are appropriately investigated and resolved. The study was conducted in accordance with the Declaration of Helsinki (as revised in 2013), and written informed consent was signed by each subject. The study protocol was approved by the Ethics Committee of West China Hospital (No. 2019: 372).

Open Access Statement: This is an Open Access article distributed in accordance with the Creative Commons Attribution-NonCommercial-NoDerivs 4.0 International License (CC BY-NC-ND 4.0), which permits the noncommercial replication and distribution of the article with the strict proviso that no changes or edits are made and the original work is properly cited (including links to both the formal publication through the relevant DOI and the license). See: https://creativecommons.org/licenses/by-nc-nd/4.0/.

\section{References}

1. Sander JW, Shorvon SD. Epidemiology of the epilepsies. J Neurol Neurosurg Psychiatry 1996;61:433-43.

2. Kwan P, Brodie MJ. Early identification of refractory epilepsy. N Engl J Med 2000;342:314-9.

3. Englot DJ, Konrad PE, Morgan VL. Regional and global connectivity disturbances in focal epilepsy, related neurocognitive sequelae, and potential mechanistic underpinnings. Epilepsia 2016;57:1546-57.

4. Berg AT, Scheffer IE. New concepts in classification of the epilepsies: entering the 21st century. Epilepsia 2011;52:1058-62.

5. Englot DJ, Hinkley LB, Kort NS, et al. Global and regional functional connectivity maps of neural oscillations in focal epilepsy. Brain 2015;138:2249-62.

6. Liu W, Yue Q, Tian Y, et al. Neural functional connectivity in patients with periventricular nodular heterotopiamediated epilepsy. Epilepsy Res 2021;170:106548.

7. Biswal B, Yetkin FZ, Haughton VM, et al. Functional connectivity in the motor cortex of resting human brain using echo-planar MRI. Magn Reson Med 1995;34:537-41.

8. Bertram EH. Extratemporal lobe circuits in temporal lobe epilepsy. Epilepsy Behav 2014;38:13-8.

9. Liu $\mathrm{W}$, Yue $\mathrm{Q}, \mathrm{Wu} \mathrm{X}$, et al. Abnormal blood oxygen level-dependent fluctuations and remote connectivity in sleep-related hypermotor epilepsy. Acta Neurol Scand. 2021;143:514-20.

10. Robinson LF, He X, Barnett P, et al. The Temporal Instability of Resting State Network Connectivity in Intractable Epilepsy. Hum Brain Mapp 2017;38:528-40.

11. Zhang Z, Lu G, Zhong Y, et al. Altered spontaneous neuronal activity of the default-mode network in mesial 
temporal lobe epilepsy. Brain Res 2010;1323:152-60.

12. Noe K, Sulc V, Wong-Kisiel L, et al. Long-term outcomes after nonlesional extratemporal lobe epilepsy surgery. JAMA Neurol 2013;70:1003-8.

13. Fahoum F, Lopes R, Pittau F, et al. Widespread epileptic networks in focal epilepsies: EEG-fMRI study. Epilepsia 2012;53:1618-27.

14. Blumcke I, Spreafico R, Haaker G, et al. Histopathological Findings in Brain Tissue Obtained during Epilepsy Surgery. N Engl J Med 2017;377:1648-56.

15. Liu $W$, Lin $M$, Yue Q, et al. Brain functional connectivity patterns in focal cortical dysplasia related epilepsy. Seizure 2021;87:1-6.

16. Hong SJ, Bernhardt BC, Schrader DS, et al. Wholebrain MRI phenotyping in dysplasia-related frontal lobe epilepsy. Neurology 2016;86:643-50.

17. Engel J Jr, Thompson PM, Stern JM, Staba RJ, Bragin A, Mody I. Connectomics and epilepsy. Curr Opin Neurol 2013;26:186-94.

18. Proposal for revised classification of epilepsies and epileptic syndromes. Commission on Classification and Terminology of the International League Against Epilepsy. Epilepsia 1989;30:389-99.

19. Kwan P, Arzimanoglou A, Berg AT, et al. Definition of drug resistant epilepsy: consensus proposal by the ad hoc Task Force of the ILAE Commission on Therapeutic Strategies. Epilepsia 2010;51:1069-77.

20. Pressl C, Brandner P, Schaffelhofer S, et al. Resting state functional connectivity patterns associated with pharmacological treatment resistance in temporal lobe epilepsy. Epilepsy Res 2019;149:37-43.

21. Koepp MJ. Neuroimaging of drug resistance in epilepsy. Curr Opin Neurol 2014;27:192-8.

22. Berg AT, Berkovic SF, Brodie MJ, et al. Revised terminology and concepts for organization of seizures and epilepsies: report of the ILAE Commission on Classification and Terminology, 2005-2009. Epilepsia 2010;51:676-85.

23. Bernasconi A. Connectome-based models of the epileptogenic network: a step towards epileptomics? Brain 2017;140:2525-7.

Cite this article as: Liu W, Yue Q, Gong Q, Zhou D, Wu X. Regional and remote connectivity patterns in focal extratemporal lobe epilepsy. Ann Transl Med 2021;9(14):1128. doi: 10.21037/atm-21-1374
24. Braakman HM, Vaessen MJ, Jansen JF, et al. Frontal lobe connectivity and cognitive impairment in pediatric frontal lobe epilepsy. Epilepsia 2013;54:446-54.

25. Cao X, Qian Z, Xu Q, et al. Altered intrinsic connectivity networks in frontal lobe epilepsy: a resting-state fMRI study. Comput Math Methods Med 2014;2014:864979.

26. Klugah-Brown B, Luo C, Peng R, et al. Altered structural and causal connectivity in frontal lobe epilepsy. BMC Neurol 2019;19:70.

27. Dong L, Li H, He Z, et al. Altered local spontaneous activity in frontal lobe epilepsy: a resting-state functional magnetic resonance imaging study. Brain Behav 2016;6:e00555.

28. Fedi M, Berkovic SF, Scheffer IE, et al. Reduced striatal D1 receptor binding in autosomal dominant nocturnal frontal lobe epilepsy. Neurology 2008;71:795-8.

29. Picard F, Bruel D, Servent D, et al. Alteration of the in vivo nicotinic receptor density in ADNFLE patients: a PET study. Brain 2006;129:2047-60.

30. Klugah-Brown B, Luo C, He H, et al. Altered Dynamic Functional Network Connectivity in Frontal Lobe Epilepsy. Brain Topogr 2019;32:394-404.

31. Scheperjans F, Eickhoff SB, Hömke L, et al. Probabilistic maps, morphometry, and variability of cytoarchitectonic areas in the human superior parietal cortex. Cereb Cortex 2008;18:2141-57.

32. Mohan A, Roberto AJ, Mohan A, et al. The Significance of the Default Mode Network (DMN) in Neurological and Neuropsychiatric Disorders: A Review. Yale J Biol Med 2016;89:49-57.

33. Kay BP, DiFrancesco MW, Privitera MD, et al. Reduced default mode network connectivity in treatment-resistant idiopathic generalized epilepsy. Epilepsia 2013;54:461-70.

34. Voets NL, Beckmann CF, Cole DM, et al. Structural substrates for resting network disruption in temporal lobe epilepsy. Brain 2012;135:2350-7.

35. Luo C, Li Q, Lai Y, et al. Altered functional connectivity in default mode network in absence epilepsy: a restingstate fMRI study. Hum Brain Mapp 2011;32:438-49.

(English Language Editors: B. Draper and J. Chapnick) 